\title{
33. COMMISSION DE LA STRUCTURE ET DE LA DYNAMIQUE DU SYSTEME GALACTIQUE
}

Président: A. Blaauw.

Membres: MM. Agekian, Ambartsumian, Baade, R. H. Baker, Bidelman, Blanco, Bok, Bourgeois, Camm, Delhaye, Durand, Dziewulski, Elvius, Finlay-Freundlich, Fricke, Habibulin, Heckmann, Hertzsprung, Ikaunieks, Jaschek, Junkes, Kaburaki, Kuiper, Kukarkin, Kuzmin, Lindblad, Lindsay, Lundmark $\uparrow$, Lyttkens, McCuskey, MacRae, Malmquist, Mergentaler, F. D. Miller, Mohr, W. W. Morgan, Nassau, Neyman, Nordström, Odgers, Ogorodnikov, Oort, Pannekoek, Parenago, Perek, Ramberg, Reiz, Mlle Roman, MM. Safronov, Schalén, Schilt, M. Schmidt, Schürer, Smart, Trumpler $\uparrow$, Vanderlinden, van Hoof, van Rhijn, Vashakidzef, Vyssotsky, Weaver.

\section{3a. SOUS-COMMISSION DE COORDINATION DES RECHERCHES GALACTIQUES}

Président: J. H. Oort.

Membres: MM. Baade, Blaauw, Lindblad, Parenago, Thackeray, van Rhijn.

\section{3b. SOUS-COMMISSION POUR LA DÉTERMINATION DU PÔLE GALACTIQUE ET DES LONGITUDES GALACTIQUES}

Membres: MM. Blaauw, Pawsey, Westerhout.

\section{INTRODUCTION}

In preparing the present report, the author, like his predecessors, has felt the impossibility of covering all aspects of the wide field of research connected with galactic problems. I have therefore excluded almost all investigations which may reasonably be expected to be reported in other commissions, such as those dealing with star clusters and associations, interstellar matter, radio-astronomical observations and various kinds of observational programmes.

Even so, a selection had to be made among the many interesting reports received from members of the commission. Moreover, a large number of important investigations have been carried out during the last few years by astronomers not belonging to the commission. It has clearly become impossible to embrace all workers in this field by one single commission; this fact, and the general policy of the Executive Committee of the Union, operate strongly in favour of a considerable reduction of the membership. Commission 33 would then have the character of a small, but probably more efficient, organizational group, which might assume some of the tasks now delegated to its sub-commissions.

\section{NUCLEUS AND HALO}

A number of investigations (Priester [r]; Hagen, Lilley and McClain [2]; Smith, O'Brien and Baldwin [3]; Westerhout [4]) deal with radio observations of the galactic nucleus and, more specifically, with the question of its identification with the radio source Sgr A. The most convincing evidence in favour of this identification is the observation of the $2 \mathrm{I}-\mathrm{cm}$ absorption at the position of this source by one of the arms discovered near the galactic centre by van Woerden, Rougoor and Oort [5].

An optical study of the central regions of the Galaxy based on infra-red photography was made by Dufay, Bigay and Berthier [6]. It disclosed an intense and irregular cloud, which probably must be identified with the nuclear region of the Galaxy. The distribution 


\section{COMMISSION 33}

of galactic novae is discussed in ch. 2 of C. Payne-Gaposchkin's monograph The Galactic Novae (North Holland Publ. Co., Amsterdam, r957).

For a description of radio observations dealing with the halo we refer to the report on radio astronomy. So far, there has been practically no common ground between the optical and radio observations of the halo. Luyten and Carpenter [7] reported on a systematic survey of faint blue stars which has been extended by Luyten and Seyfert [8]. Colour indices and proper motions were measured for stars found on Tucson plates in 45 Selected Areas. They appear to be objects of intermediate luminosity, not fainter than +5 absolute magnitude; surprisingly few white dwarfs have turned up in the survey. Another extensive survey of blue stars in high galactic latitudes is being carried out at Tonantzintla (Iriarte and Chavira) [9]. Surveys of variable stars have been inaugurated by Shapley [10] and by the Kapteyn Laboratory at Groningen in collaboration with the Mount Wilson and Palomar observatories. For details concerning the latter project we refer to the report on the Stockholm conference on Co-ordination of Galactic Research (see below). The aim of the Groningen-Palomar programme is the determination of the shape of the equi-density surfaces of the population of variable stars in the halo. This programme is being supplemented by an objective-prism survey of late-type variable stars by Blanco at Cleveland, based on plates taken at the Nassau station of the Case Observatory and reaching I9.5 photographic magnitude.

\section{SPIRAL STRUCTURE}

\section{Radio observations}

Since the previous report of this commission, various important $2 \mathrm{I}-\mathrm{cm}$ surveys of the distribution of neutral interstellar hydrogen have been brought to a provisional completion. The southern sky has been covered by the Australian observers, and the northern observers have extended their surveys to the inner parts of the Galaxy. Of these latter, one published by Schmidt [ri] covers longitudes $340^{\circ}$ to $50^{\circ}$ and shows that the Orion and Sagittarius arms can be traced throughout this inner region; both arms appear to be trailing and seem to be connected by a hydrogen bridge near the Sun. Schmidt suggests that this bridge, between $l=340^{\circ}$ and $20^{\circ}$, explains the large difference between the small inclination of the spiral arms as found from the radio observations and the larger value derived from the positions of $\mathrm{O}$-associations.

The hydrogen in the inner parts of the Galaxy is found to be confined to a very thin layer of only $220 \mathrm{pc}$ thickness between half-density planes. The average density between 6 and $8 \mathrm{kpc}$ distance from the centre is $0.85 \mathrm{H}$ atoms per cc, and, between 4 and $6 \mathrm{kpc}$, $0.6 \mathrm{H}$ atoms per cc.

Results of the Australian survey have been published by Kerr, Hindman and Carpenter [12] and by Kerr and Hindman [13]. The multi-armed structure of the Galaxy is confirmed as well as the trailing tendency of the arms, and there are suggestions that the major arms can be followed through several revolutions. The question of possible systematic deviations from circular motion, which is important because circular motions are assumed in the determination of the distances of the neutral hydrogen, has been given due attention. The consequences of possible deviations were, among others, discussed by Edmondson [14], but according to the Australian investigators there appears no evidence for them. Asymmetries in the velocity pattern about the galactic centre are ascribed to local disturbances.

The remarkable flatness of the gas layer in the inner parts of the Galaxy was confirmed by the Australian survey. In the outer regions the gas layer is found to show a systematic distortion, twisting to negative $z$ values on one side of the Galaxy, and to positive $z$ values at the opposite side. A connexion with the gravitational force due to the Magellanic Clouds has been suggested, but the observed effect is much larger than that to be expected from the Clouds.

Integrated over the whole Galaxy, the proportion of neutral hydrogen is only $2 \%$ of 


\section{STRUCTURE DU SYSTEME GALACTIQUE}

the total mass. This ratio increases from the centre outwards, reaching about $15 \%$ in the solar neighbourhood.

The earlier investigations of the overall properties of the hydrogen distribution by the Leiden observers have been followed by more detailed work on the nuclear region and on the distribution perpendicular to the galactic plane. Van Woerden, Rougoor and Oort [s] have found that the central regions not only exhibit the large velocity dispersion, but that in the region within $2 \mathrm{kpc}$ spiral arms occur, moving away from the nucleus with velocities between 50 and $100 \mathrm{~km} / \mathrm{sec}$. Westerhout [15] has derived the three-dimensional picture of the distribution of neutral hydrogen beyond $8.2 \mathrm{kpc}$ from the centre in the longitude interval $340^{\circ}$ to $220^{\circ}$. Diagrams are given for the cross sections through the spiral structure at intervals of $2: 5$ in longitude. The Orion arm and the Perseus arm are the major features, but faint outer arms are suggested, extending as far as $15 \mathrm{kpc}$ from the galactic centre. Detailed investigations of the local distribution of neutral hydrogen have also been made by the Harvard observers Lilley [16], Heeschen [17] and Davis [18], and by Helfer and Tatel [rg].

The Dutch $2 \mathrm{I}-\mathrm{cm}$ observations were also analysed by Parenago [20]. Except for an additional inner spiral structure, the distribution of the neutral $\mathrm{H}$ was confirmed. Application of the formula for the angular velocity,

$$
\omega^{-1}=X+Y R^{2}
$$

which has been found to fit the observations satisfactorily, gives among other results, $A /(-B)=\mathrm{I} \cdot 53 \pm 0.07$ and $R_{0}(A-B)=233 \mathrm{~km} / \mathrm{sec} \pm 6 \cdot 5$.

\section{Optical observations}

Important material for the optical study of the spiral structure of $O$ and $B$ stars is contained in Morgan, Code and Whitford's [21] list of photometry and luminosity classifications of 1270 blue giant stars, in Hiltner's [22] list of 1260 stars and in Miss Hoffleit's [23] list of spectral classes and photometry of 150 southern stars. These lists will be of basic importance for future more refined narrow-band photometry for the purpose of exact distance determinations. A most interesting study of the spiral structure, based on the displacements and intensities of interstellar $\mathrm{Ca}$ II and $\mathrm{Na}$ I absorption lines in the spectra of II2 stars with longitudes between $55^{\circ}$ and $160^{\circ}$ was published by Münch [24]. Two strong components are observed for stars beyond $2 \mathrm{kpc}$ with relative separations reaching a maximum of $30 \mathrm{~km} / \mathrm{sec}$ around $l=100^{\circ}$, and it is inferred that the interstellar matter producing these lines is arranged along the spiral arms identified by earlier investigations.

Kurochkin [25], using data on colours in Selected Areas, has found a tendency for the absorbing matter to be distributed along the spiral structure. This was also inferred from colour excesses of $\mathrm{O}$ - and B-type stars by Kiang [26].

\section{GALACTIC ROTATION}

Various investigations have been made of the effect of differential galactic rotation. Important new observational data for such studies are the radial velocities of fifty-five southern cepheids determined by Stibbs [27] and those of several hundreds of southern B stars published by Feast, Thackeray and Wesselink [28].

Analyses of the Cepheid radial velocities were carried out by Stibbs[29] and by Gascoigne and Eggen [3०]. The values of the constant $A$ found by these authors, from northern and southern cepheids, $I 9.5 \mathrm{~km} / \mathrm{sec} \pm I \cdot 9$ and $I 7.5 \mathrm{~km} / \mathrm{sec} \pm I .9$ respectively, are in good agreement ; since Gascoigne's and Eggen's determination incorporates somewhat more exact photometric data their result may deserve somewhat larger weight. Other determinations of the constant $A$ of galactic rotation are those by Petrie, Cuttle and Andrews [31] and by Weaver [32]. The first mentioned authors' result, $A=\mathrm{I} 7 \cdot 7 \mathrm{~km} / \mathrm{sec}$ $\pm I \cdot I$ is based on radial velocities of B-type stars for which spectroscopic absolute magnitudes had been determined at Victoria. 
Weaver has critically discussed the significance of the results for solutions of the constants of rotation obtained according to the generally applied procedures. According to Weaver, the linear equation for the effect in the radial velocity produces a numerically too large $A$ value, except when applied to a sample population in the immediate solar neighbourhood. The amount of the error introduced by the use of the linearized theory has been checked by Weaver by a number of tests, assuming models in which objects over large distances from the Sun are included in the analysis. The value of $A$ in Weaver's discussion is defined by the slope of the $\omega(R)$ curve at the Sun's distance from the galactic centre. From investigations of cepheids and B stars Weaver arrives at 'unbiased 'values of $A$ of II and I $3 \mathrm{~km} / \mathrm{sec}$ per $\mathrm{kpc}$, considerably lower than the previously adopted values which lie between 15 and $20 \mathrm{~km} / \mathrm{sec}$ per $\mathrm{kpc}$.

Although the above mentioned investigations by other authors, which took account of Weaver's remarks, have not confirmed the very low value of $A$, it is clear that considerable caution should be exercised in the definition and use of the constants $A$ and $B$. This is the more important because values of these constants are involved in determinations of the distance of the galactic centre and of the velocity of rotation of the Galaxy. It has been shown by Weaver that the difference $A-B$, which is equal to the angular velocity of rotation, is free from possible bias in the procedure followed in determining the constants $A$ and $B$ from a given sample of stars. Determinations of this quantity give a mean value of about $25 \mathrm{~km} / \mathrm{sec}$ per kpc. Another important quantity, derived from $2 \mathrm{I}-\mathrm{cm}$ observations, is the product $A R_{0}, R_{0}$ being the distance of the Sun from the galactic centre. For this, Schmidt finds $152 \mathrm{~km} / \mathrm{sec}$ per kpc \pm Io. With a value of $A$ of $\mathrm{I} 8 \mathrm{~km} / \mathrm{sec}$ per $\mathrm{kpc}$ this gives $R_{0}$ between 8 and $9 \mathrm{kpc}$. This is in good agreement with Baade's value of $8.2 \mathrm{kpc}$ derived from the statistics of short-period cluster-type cepheids in the nuclear region of the Galaxy.

Yet, it cannot be said that the present knowledge of the distance of the galactic centre, and, more generally, of the galactic distance scale, is satisfactory. Questions such as the following are still open: what is the exact value of the absolute magnitudes of the clustertype variables in the galactic nuclear regions? how well do we know the interstellar absorption in this direction? to what extent are the values of $A$ and $B$ determined by stars in the solar neighbourhood representative for the regions in general at the Sun's distance from the centre? A concerted attack on problems covering all aspects of the determination of the galactic centre is urged by Weaver, and would seem to deserve the attention of workers in various fields within this commission. Of various current investigations related with this general problem, we mention a determination of the constant $B$ from Yale catalogues by Mohr, Mayer and Stohl [33] and current work by Mohr and Mayer on the dependence of the rotational velocity on the distance from the galactic centre; an investigation by Schmidt of the correction to the constants $A$ and $B$ required by the presence of the Orion arm, and of the consequences of the use of modified values of $A, B$ and $R_{0}$ for his calculated model of the mass distribution in the Galaxy (see also below); and work on the zero-point of the period-luminosity function for longperiod cepheids by Pskovsky [34] who finds a correction of $-I \cdot 3$ magnitude to Shapley's zero-point. A determination by Gascoigne and Eggen [30] gave $-\mathrm{x} \cdot 7$ for this correction.

\section{PROPERTIES OF THE LOCAL POPULATION}

\section{Bright stars}

A number of lists of accurate observational data for spectral classifications and photometry are very useful for the study of the local properties of the Galaxy. Spectral and luminosity classifications along the lines of the Yerkes system developed by Morgan and associates were published by A. de Vaucouleurs [35] for 366 B-, A- and F-type southern stars brighter than magnitude 6.5 , by Bidelman [36] for $24 \mathrm{I}$ stars known as possible $F, G$ or $\mathrm{K}$ super-giants, by Halliday [37] for $227 \mathrm{G}$ and $\mathrm{K}$ stars of the David Dunlap radial velocity programmes, and by Miss Roman [38] for 600 high-velocity stars. Luminosity 


\section{STRUCTURE DU SYSTEME GALACTIQUE}

criteria applicable to low-dispersion infra-red slit spectrograms of M-type stars were discussed by Sharpless [39]. The need for a spectral and luminosity classification based on purely photometric information has led to a number of different approaches. We mention the very precise photographic spectrophotometry by Chalonge and associates [40], the narrow-band photo-electric photometry developed by Strömgren and associates [4x], the measurements of $\mathrm{H} \gamma$ as an indicator of absolute magnitude in early-type stars by Petrie [42] and of $\mathrm{H} \beta, \mathrm{H} \gamma$ and $\mathrm{H} \delta$ by Stock [43]. Finally, O. C. Wilson and Vainu Bappu [44] have demonstrated that one of the most promising methods for the determination of the luminosities of $G, K$ and $M$ stars appears to be the measurement of the width of the emission reversal in the $\mathrm{H}$ and $\mathrm{K}$ lines. Spectrophotometric criteria for three-dimensional classification in small dispersion are investigated at the Torún Observatory (Poland) with some positive results obtained for late-type stars.

A few of the many interesting discussions of the properties of the brighter stars may be summarized here. Miss Roman, on the basis of the data just mentioned, supplemented by accurate photometry in the $U, B, V$ system, found that the H.R. diagram of the highvelocity stars is similar to that of the old galactic cluster M 67 and not to that of globularcluster stars. She was also able to show that a very good correlation exists between the ultra-violet excess and the space velocity for stars with $B-V$ between $+0 \cdot 3$ and $+\mathbf{r} \cdot \mathrm{I}$. Halliday's absolute magnitude classifications have led to a new determination of the luminosity function and space motions of G 8-K I stars; the problem of the calibration of the luminosity classification remains a nasty one, however. Petrie's luminosities for early-type stars have provided a new basis for the discussion of the motions and distributions in space of these objects.

\section{The nearest stars}

A catalogue of $9 I_{5}$ stars within 20 pc was compiled by Gliese [45], who also studied [46] various properties of this sample of the galactic population. The mass density is found to be 0.057 solar masses per cubic pc or $3.9 \times 10^{-24} \mathrm{~g} / \mathrm{cm}^{3}$, and the ratio mass/luminosity $2 \cdot 5$. The cosmical scatter with respect to the main sequence of the $\mathrm{G} \mathrm{o-M} 7$ stars with space velocities below $60 \mathrm{~km} / \mathrm{sec}$ is \pm 0.2 magnitudes. The scatter is found to increase with the space velocities of the stars, and the high-velocity stars are on the average 0.24 magnitude below the main sequence. A study of the space velocities shows concentrations around the velocities of the Ursa Major stream and the Taurus stream.

Eggen [47] made a photometric study of 275 bright stars with trigonometric parallaxes greater than $0^{\prime \prime} .02$, with particular reference to the populations in different parts of the H.R. diagram. The interesting question arises whether sub-giants exist in the solar neighbourhood that may be older than $M 67$ or that may have undergone an evolution different from the usual course for the giant stars. Photometry for seventy-seven bright nearby stars was published by Johnson and Knuckles [48]. Red and infra-red magnitudes for 282 nearby stars were measured by Kron, Gascoigne and White [49].

\section{Kinematics of nearer stars}

The motions and distributions of dwarf M stars, discovered spectro-photometrically at the McCormick Observatory, were studied by Mumford [50], and the space motions of red-dwarf stars, mostly $\mathrm{K} 8$ to $\mathrm{M} 2$, by Dyer [5r]. The velocity distribution of nearby dwarfs was also studied by A. W. Wehlau [52]. In these studies, particular care is taken to eliminate selection effects in the material. Among other investigations of the velocity distribution of the stars in the solar neighbourhood we mention those by Einasto [53], dealing with a method of computing the velocity dispersion which he applied to the emission M dwarfs, and by Nahon [54], who gave a new method for the derivation of Ambartsumian's formula for the analysis of space velocities based on radial velocities and applied this to $\mathrm{A}$ and $\mathrm{K}$ stars. A card file containing data on space motions, positions and other relevant data is being compiled by Rigal at the Institut d'Astrophysique. The relation between stream and ellipsoidal constants was studied by Ewart [55]. 


\section{COMMISSION 33}

Ogorodnikov has studied the relation between the $K$ effect and the anomalies in the velocity ellipsoid of $B$ stars, and the dynamical instability of this system of stars.

There would not be much point in describing the detailed results obtained in these investigations without reference to the extensive preceding research in this field. A review of problems connected with the study of the solar motion and the distribution of peculiar motions has been given by Vyssotsky [56]. Two points may be briefly referred to here.

(I) The McCormick investigators have introduced the concept of 'basic solar motion', which is the solar motion with respect to 'the most probable velocity' as defined by the greatest concentration of velocity-vector points, and which, according to Vyssotsky and associates, also refers to the circular velocity with respect to the galactic centre. This basic solar motion according to Dyer [5r] is $15.3 \mathrm{~km} / \mathrm{sec} \pm 0.2$ directed toward $A=262^{\circ} 4, D=+20^{\circ} 3$ or $l=\mathrm{II}{ }^{\circ} \mathrm{O}, b=+24^{\circ} 4$. According to Edmondson [57], the difference between this solar motion and higher values of the velocity obtained from distant objects (Cepheids, OB stars, interstellar $\mathrm{Ca}^{+}$) is due to incomplete elimination of the effect of differential galactic rotation in the latter investigations.

(2) It is becoming increasingly evident that investigations of stellar motions yield significant results only if the various population groups are discriminated according to spectroscopic or photometric characteristics. Thus, Vyssotsky [56] has demonstrated that the vertex deviation is confined to stars moving close to the galactic plane, whereas it vanishes for stars with large orbital inclinations, and that this distinction can be reduced to a separation of the stars according to spectroscopic criteria identified among $F$ and $\mathrm{G}$ dwarfs, $\mathrm{G} 8$ to $\mathrm{K} 2$ giants and $\mathrm{M}$ dwarfs. Detailed investigations of the structure of vector-point distributions suggest the existence of discrete streamings, the study of which for various types of stars may be an interesting tool connecting kinematical properties and evolutionary stages.

\section{Spectral surveys; studies of space distribution}

From various institutes spectral surveys are reported which form the basis for statistical investigations. Malmquist reports a spectro-photometric survey combined with photoelectric photometry along the lines of earlier work [58] for the Milky Way in the intervals of galactic longitude $40^{\circ}$ to $60^{\circ}, 10^{\circ}$ to $40^{\circ}$ and $80^{\circ}$ to $110^{\circ}$, for stars brighter than photographic magnitude 9.5 . Malmquist also reports the determination of spectral types and luminosity classes for about 2500 stars down to photographic magnitude 13.5 in the region of the north galactic pole, based on Stockholm plates. A similar investigation for the south galactic pole, based on plates taken in Australia, is in progress. Ljunggren, at Uppsala, determines photo-electric colours and magnitudes for early-type stars near the north galactic pole from Malmquist's earlier surveys, one of the aims being the determination of intrinsic colours for the various spectral and luminosity classes. A spectroscopic classification programme for all stars as early as A 2 in the Henry Draper Catalogue within $12^{\circ} \cdot 5$ of the north and south galactic poles is being carried out by Bidelman at the Lick Observatory.

A spectral survey of the entire sky north of $+25^{\circ}$ declination on objective-prism plates taken with the Hamburg Schmidt telescope is in progress (dispersion $580 \AA / \mathrm{mm}$ at $\mathrm{H} \gamma$ ). Stars from the $7^{\text {th }}$ to the $13^{\text {th }}$ magnitude can be classified. Priority is being given to the Milky Way areas and the zone between $+25^{\circ}$ and $+30^{\circ}$ declination, and a search for OB stars and A-, F- and G-type super-giants has been started by Slettebak and Stock. On the latter programme, the region between $0^{\mathrm{h}}$ and $2^{\mathrm{h}} 40^{\mathrm{m}}$ in right ascension has been catalogued. Stars earlier than $\mathrm{F}$ o in an area approximately 500 square degrees around the north galactic pole are searched for by Slettebak.

At the Stockholm Observatory, Ramberg has continued his photometric and spectrophotometric survey of selected regions in and near the Milky Way, the primary aim being the determination of space densities for various spectral classes. The survey for the Lacerta region $\left(l=69^{\circ}, b=-8^{\circ}\right)$ down to photographic magnitude $13 \cdot 2$ has been completed [59]. From the data for 3000 stars Ramberg finds a cloud of heavy absorption and 


\section{STRUCTURE DU SYSTEME GALACTIQUE}

increased star density, located between 900 and $1500 \mathrm{pc}$, coinciding with the Orion arm of neutral hydrogen. An extension of the survey in the near infra-red regions is in preparation. Four selected fields in Aquila-Sagitta and Cassiopeia are being investigated by similar methods at the Torún Observatory (Poland). Two of these fields in Sagitta and Cassiopeia have been analysed for the distribution of stars and absorbing matter by C. Iwaniszewska and R. Ampel.

Bok [60] has analysed star counts on Palomar-Schmidt plates for heavily obscured areas in Ophiuchus and in Taurus. The penetrating power of this material appears sufficient to estimate photographic absorption up to 8 magnitudes in features larger than 0.3 degrees across. The same author [6r] has discussed the Milky Way structure in Sagittarius, $l=325^{\circ}$ to $330^{\circ}, b=0^{\circ}$ to $-10^{\circ}$ and in Carina, $l=250^{\circ}$ to $263^{\circ}, b=-5^{\circ}$ to $+5^{\circ}$, with emphasis on the different character of these two regions. The Sagittarius cloud represents preponderantly old population, whereas the Carina section is a pronouncedly young population region.

The Leander McCormick Observatory has undertaken to classify the spectra of the AGK 2 stars not previously classified elsewhere. The first part, covering the declination zones $+50^{\circ}$ to $+85^{\circ}$ has been published [62]. The second part $\left(-2^{\circ}\right.$ to $\left.+50^{\circ}\right)$ is in progress.

Since the last report, the survey of the infra-red spectra of low temperature stars in the Milky Way at the Warner and Swasey Observatory has been completed. A zone of $\mathrm{I} 2^{\circ}$ wide from $l=333^{\circ}$ to $l=201^{\circ}$ has been covered down to infra-red magnitude I0.0. A second list of $\mathrm{M}_{5}$ to $\mathrm{M}$ Io $\mathrm{BD}$ stars has been published [63], while one of the $\mathrm{M} 2$ to $\mathrm{M} 4 \mathrm{BD}$ stars is in preparation. A new catalogue of early $M$ stars with abnormal reddening, containing a high proportion of distant super-giants has also been completed [64]. Whereas the distribution of the late $\mathrm{M}$ stars corresponds to that of a uniformly distributed disk population, the distribution of the carbon stars reveals definite groupings. A similar conclusion was reached by E. and H. Smith [65], who studied the southern Milky Way by means of $\mathrm{ADH}$ plates. A second catalogue of the carbon stars based on the Cleveland surveys was published by Nassau and Blanco [66]. More work on these stars is in progress. The extension of the survey for $S$ stars in the zones $\pm 2^{\circ}$ to $\pm 6^{\circ}$ was completed by Blanco and Nassau [64]. These stars, like the highly reddened early $M$ stars, are strongly concentrated toward the galactic plane. The M-star distribution in the Cleveland survey has been studied by Sanduleak [67]. An examination of the possible interrelation of lowtemperature stars with the blue and the F super-giants is also in progress at Cleveland.

A survey of M super-giants, based on plates taken with the Hamburg original Schmidt telescope, is being carried out at the Astronomisches Rechen-Institut. Velghe [68], using $\mathrm{ADH}$ plates, demonstrated the applicability of the yellow-red region of low-dispersion spectra by studying regions in Sagittarius and in Vela. Van Rhijn [69], from studies of the space distribution of the A o to $A_{5}$ stars and $K$ giants near the Sun, finds that the space densities of the A-type stars within $50 \mathrm{pc}$ from the galactic plane are correlated with the hydrogen densities within the same layer, whereas no such correlation exists for the $\mathrm{K}$ giants. The correlation vanishes for distances beyond $50 \mathrm{pc}$ from the plane. The points of maximum space density of the A-type stars and of the $\mathrm{K}$ giants do not seem to coincide with the OB-star aggregates.

\section{Force and Motions Perpendicular to the Galactic Plane}

Oort reports a new determination of $K_{z}$ by Hill, based on counts of $\mathrm{K}$ stars in high latitudes, combined with the distribution of proper motions of these stars. The $\mathrm{K}$ stars were counted in the Henry Draper Catalogue and, for the fainter stars, in the Bergedorfer Spektral-Durchmusterung. The proper motions served to determine the fractions of giants. An extensive discussion was made of the distribution of linear velocities. Combining Hill's data with the working hypothesis that the principal part of $K_{z}$ is due to interstellar matter and to stars distributed in approximately the same manner as the $\mathrm{K}$ giants, Oort derived a value of $\mathrm{I} \cdot 0 \times \mathrm{IO}^{-23} \mathrm{~g} / \mathrm{cm}^{3}$, or $0 \cdot I_{5}$ solar masses per cubic parsec, for the total density of matter at $z=0$ in the general vicinity of the Sun. 


\section{COMMISSION 33}

Another investigation of the force perpendicular to the galactic plane and of the stellar motions in this component was published by Nahon [7o], who finds indications of a larger mass density near the galactic plane than was found originally by Oort, and an increase of the velocity dispersion with the distance from the galactic plane. The results are, however, admittedly uncertain and the importance of a more accurate determination of the velocity distribution for stars of various luminosity classes is stressed. Woolley [7r], from a new discussion of the motions and density distribution of A-type stars, finds evidence for a total density near the Sun as high as $0 \cdot 18$ solar masses per cubic pc. The lower value of 0.08 solar masses per cubic pc is obtained in a discussion by Kuzmin [72], and a criticism of work by other investigators has been given by Eelsalu [73]. The dependence of the mean speed of the stars in the direction perpendicular to the galactic plane as a function of the distance from the plane has been investigated by Panaiotov [74].

\section{Luminosity functions}

McCuskey's study [75] of the luminosity function in eleven Milky Way regions, based on new spectral, magnitude and colour data, has been completed. The average luminosity function derived for all longitudes and distances within $500 \mathrm{pc}$ is found to agree well with the standard Van Rhijn function. The maximum deviations at certain locations are 0.6 in $\log \phi(M)$. In addition, results on the galactic structure within Iooo pc are given: for the B 5-A o stars even up to distances of $2500 \mathrm{pc}$. Studies of the luminosity function are also being made at the La Plata Observatory by Wilkens who, from an analysis of star counts, finds evidence for an abrupt change in the luminosity law at $z=100 \mathrm{pc}$, ascribed to an excess of low luminosity objects at low $z$. Investigations of the absolute magnitudes of the metallic-line stars and the peculiar A stars are reported by Jaschek.

\section{Motions and space distributions of special groups of stars}

A large number of investigations of stars of certain types have been completed or are in progress, many of which may lead to an eventual understanding of the role of these stars in the evolutionary scheme of the Galaxy.

Improved proper motions of 650 bright B-type stars were published by H. R. Morgan [76]. Filin [77] studied the kinematics of B stars with special reference to their membership of a local system. The luminosities, space distribution and motions of the nearer northern O-B 5 stars were investigated by Blaauw [78]. The relation of special types of stars ( $\mathrm{M}$ dwarfs, $\mathrm{S}$ stars) to clusters is being investigated by Blanco and Grant as part of an extensive programme of clusters. Daube [79] studied the space distribution and kinematics of 476 spectroscopic binaries of different types and found no difference with respect to the single stars. On the other hand, Blaauw [8०] and Wolterbeek Muller (Leiden) found larger space velocities for single stars than for double stars.

Proper motions of 337 long-period variables have been measured at the McCormick Observatory under the supervision of Alden and Osvalds, and for many of these the radial velocities will also become available. The kinematics of the sub-system of the long-period Mira-Ceti variables was studied by Safronov [81]. Joy [82] has added radial velocities of I5 RR Lyrae variables to his two previous lists. Oosterhoff [83], from a discussion of 129 known classical cepheids in a region of 5000 square degrees in the direction of the galactic centre, has found strong evidence that at least twenty-five of the brightest variables must belong to population I, and more than seventy to population Ir. Kukarkin and his collaborators are continuing their morphological studies of variable stars, with particular attention to RR Lyrae stars in globular clusters and Mira-Ceti variables in galactic fields.

Radial velocities of planetary nebulae are being determined by Mayall and Minkowski [84].

Miss Shazova [85] has continued her investigations of the observational selection of absolutely faint stars and its influence on the kinematic characteristics and the luminosity function. She finds that the faint end of the Luyten luminosity function requires a considerable increase. The white dwarfs, according to Pavlovskaja's [86] studies of their kinematics and luminosity, form an intermediate sub-system in the Galaxy. 


\section{STRUCTURE DU SYSTEME GALACTIQUE}

W. and A. Dziewulski report studies of the motions of A- and F-type stars with known parallaxes, proper motions and radial velocities which indicate the existence of a vast streaming of stars towards the direction of longitude $0^{\circ}$. W. Dziewulski has undertaken a study of the galactic rotation of giant $\mathrm{A}$ and $\mathrm{F}$ stars. These studies will be published in the Bulletin of the Torún Observatory.

Mean parallaxes for stars of various spectral types have been determined by Heintz [87] from McCormick and Cape proper motions.

\section{DYNAMICS OF THE GALAXY}

A concise treatment of the mechanics of stellar systems has appeared in R. Kurth's monograph Introduction to the Mechanics of Stellar Systems [88], and a review of recent developments in Vistas in Astronomy by Camm [89].

A model of the mass distribution in the Galaxy, based on all available optical and radio-astronomical data has been constructed by Schmidt [oo]. It is based on a particular set of values of the constants $A$ and $B$ of galactic rotation and of the distance $R_{0}$ to the galactic centre, and fits such observations as the observed absence of high-velocity stars exceeding $65 \mathrm{~km} / \mathrm{sec}$ in the direction of galactic rotation, the rotational velocity as a function of the distance from the galactic centre, and observed density gradients of certain types of stars. However, the necessity to introduce a class of objects of unknown character with a steep density gradient near the Sun has caused some doubt as to the correctness of the model and of the values of the constants adopted. According to Schmidt, the values $A=15 \mathrm{~km} / \mathrm{sec}$ per kpc, $B=-10 \mathrm{~km} / \mathrm{sec}$ per kpc and $R_{0}=10 \mathrm{kpc}$ would be sufficient to avoid this difficulty. In that case the total mass of the Galaxy would be twice the value of $7 \times 10^{10}$ solar masses found originally, and the rotational velocity near the Sun would become $250 \mathrm{~km} / \mathrm{sec}$ instead of $216 \mathrm{~km} / \mathrm{sec}$.

As an extension of Schmidt's work, Fricke, at Heidelberg, has undertaken the construction of a model of both the distribution of mass and of velocities. This includes a simultaneous solution of Boltzmann's and Poisson's equations. Attempts at finding a non-stationary distribution of mass and velocities in view of the particular problem of the evolution of globular clusters are being made by Nahon.

Kuzmin [9r] has continued his work on the theory of a model of a stationary Galaxy and worked out the case permitting a tri-axial velocity distribution. It has led, among other results, to a determination of the obliquity of the velocity ellipsoid outside the galactic plane and to a generalization of the Lindblad and Bottlinger diagrams. Kuzmin [92] has also applied a previously developed method for determining the projected density in a flattened stellar system to the $2 \mathrm{I}-\mathrm{cm}$ results. The total mass of the system is found to be II $\times 10^{10}$ solar masses and the circular velocity near the Sun $250 \mathrm{~km} / \mathrm{sec}$.

The motions of stars in an ellipsoidal stellar system were studied by Contopoulos [93] for the case of equi-density surfaces of constant axes-ratio. This author [94] also studied the relative motions of stars in a galaxy in two and in three dimensions. This study treats the problem, to what extent the 'torus section', defined by the motion of a particle on a plane passing through the axis of symmetry of the system and the particle, is actually filled by the orbit. A model of the Galaxy, with increasing flattening of the surfaces of equal density with increasing distance from the centre was developed by Mikisha and Tsitsin [95]. Current studies of Ogorodnikov deal with the statistical mechanics of stellar systems consisting of a main body surrounded by a corona, where hydro-dynamical methods are applicable only to the main body. The various possible models for the density distribution are related to different types of extra-galactic systems and an evolutionary development from needle-like systems to spirals and then to elliptical galaxies is suggested. Current studies by Agekian indicate that dissipation of stars from a rotating stellar system may result in compression of the system or in decreasing compression, depending on a critical initial flattening of the system.

The origin of the high-velocity stars was investigated by van Wijk [96], who showed that the assumption that these stars are part of the isotropic Maxwellian velocity 


\section{COMMISSION 33}

distribution, and originated at different distances from the galactic centre, may suffice to account for their existence.

Idlis [97] extended Parenago's work on the galactic potential and developed a theory of the ages of stars of types $\mathrm{O}, \mathrm{B}, \mathrm{A}$ and their velocity distributions proceeding from Schwarzschild's and Spitzer's theory of the dispersion of stellar velocities due to encounters, and from the theory of corpuscular evolution.

A thorough investigation of the possible existence of stars with hyperbolic velocities, and the related question of the velocity of escape from the Galaxy was published by Perek [98]. For at least eighteen stars the excess of the observed velocity over the velocity of escape seems to be well established. Among these are O- or B-type stars of recent formation, RR Lyrae stars, and other objects of various types. A number of objects have retrograde motions in the Galaxy. The existence of stars escaping from the Galaxy raises the most interesting question of the density of a possible inter-galactic population.

B. Lindblad [99] has further developed his theory of the orbits along which the particles of a dissolving cloud tend to disperse. This theory has a bearing on the interpretation of the vertex deviation and of differential motions in the Galaxy, and on the general problem of the spiral structure. Lindblad based his computations on the angular-velocity curve as derived from the $2 \mathrm{I}-\mathrm{cm}$ measures, and found that there are mainly two types of dispersion orbits: those with the shape of approximately Keplerian ellipses in the outer regions of the Galaxy, with stationary apsidal lines, and those in the inner regions with an elliptic shape, centred on the Galactic centre and with a rotating apsidal line. The dynamics of ring-shaped formations defined by a certain dispersion orbit are being investigated by $\mathrm{P}$. O. Lindblad with particular reference to the possible generation of spiral structure.

\section{STELLAR POPULATIONS AND EVOLUTION}

It has become more and more apparent that for studies of the evolution of the Galaxy a division of its population into more than Baade's two population groups is desirable. A division into a large number of sub-systems according to the flatness of the space distributions has been introduced several years ago by Kukarkin, mainly on the basis of studies of variable stars. A division which in some respects strikes a balance between these two, and which seems very suitable as a starting-point for investigations of the evolution of the Galaxy, has been formulated at a conference on Stellar Populations recently held under the auspices of the Vatican Academy of Sciences. For particulars concerning the proceedings at this conference we refer to the forthcoming report, edited by $\mathrm{Dr} \mathrm{D}$. O'Connell. The proposed division is given in Table I. It is a modification of two somewhat different versions due originally to Oort and to Schwarzschild. The five divisions, Halo population II, Intermediate population II, Disk population, Older population I and Extreme population I, are based mainly, but not entirely, on the degree of flatness of the space distribution. Additional characteristics, which were taken into account, are the degree of concentration towards the galactic centre, the kinematical properties and spectroscopic criteria. The five divisions form a continuous sequence and a certain degree of variation exists within each of them. It is not impossible that particularly within the middle division appreciable differences in evolutionary stages are assembled. For each division are given a number of typical kinds of objects.

The problem of the evolution of the population of the Galaxy forms the background of a large variety of investigations. Investigations of the principal contributors to the integrated light of the 'main bodies' of a number of globular clusters and galaxies of various types are reported by Morgan. They lead to the following conclusions:

(I) Globular clusters and galaxies considered to be examples of Baade's population II category appear to have very dissimilar stellar populations; globular clusters located in the galactic halo have integrated spectra different from a group in the galactic disk. In addition, integrated spectra of main bodies of the giant elliptical galaxies in the Virgo cluster are completely different from those of the halo globular clusters. The conclusion seems inescapable that the simple division of stars into two discrete populations is no longer justified. 


\section{STRUCTURE DU SYSTEME GALACTIQUE}

(2) A joint investigation by Morgan and Mayall [roo] indicates that the principal contributors to the luminosity of the inner parts of the giant ellipticals which have been observed are giant $\mathrm{K}$ stars having approximately normal metallic-line intensity; the spectra of these objects resemble closely the spectra of the inner regions of the giant $\mathrm{Sb}$ spirals similar to $\mathrm{M}$ 3I. At the other extreme, spectra of irregular galaxies similar to NGC 4449 are in general early in type, and suggest large-scale physical conditions similar to those prevailing on a smaller scale in galactic $\mathrm{O}$-associations and $\mathrm{H}$ II regions.

There is a marked correlation between galactic forms and spectra; systems having little or no central concentration of light usually have spectra of early type; those whose light has a pronounced central concentration tend to have considerably later types. The latest spectral types of the main bodies of galaxies are probably associated with giant elliptical and SBO systems; for the members of these groups which have been observed the spectral type is commonly $\mathrm{K}$, even in the ultra-violet.

Table I. Proposed divisions of stellar populations

\begin{tabular}{|c|c|c|c|c|}
\hline $\begin{array}{c}\text { Halo } \\
\text { population II }\end{array}$ & $\begin{array}{l}\text { Intermediate } \\
\text { population II }\end{array}$ & $\begin{array}{c}\text { Disk } \\
\text { population }\end{array}$ & $\begin{array}{c}\text { Older } \\
\text { population } 1\end{array}$ & $\begin{array}{c}\text { Extreme } \\
\text { population I }\end{array}$ \\
\hline Sub-dwarfs & $\begin{array}{l}\text { High-velocity } \\
\text { stars with } z \text { - } \\
\text { velocities } \\
>30 \mathrm{~km} / \mathrm{sec}\end{array}$ & $\begin{array}{l}\text { Stars of galactic } \\
\text { nucleus }\end{array}$ & A-type stars & $\begin{array}{l}\text { Gas } \\
\text { Young stars asso- } \\
\text { ciated with the } \\
\text { present spiral } \\
\text { structure }\end{array}$ \\
\hline $\begin{array}{l}\text { Globular clusters } \\
\text { of high } z \text {-motion }\end{array}$ & $\begin{array}{l}\text { Long-period } \\
\text { variables with } \\
\text { periods < } 250 \\
\text { days and spect- } \\
\text { ral types earlier } \\
\text { than M } 5\end{array}$ & $\begin{array}{l}\text { Planetary nebulae } \\
\text { Novae } \\
\text { RR Lyrae stars } \\
\text { with periods } \\
<0.4 \text { days }\end{array}$ & Strong-line stars & $\begin{array}{l}\text { Super-giants } \\
\text { Cepheids } \\
\text { T Tauri stars }\end{array}$ \\
\hline $\begin{array}{l}\text { RR Lyrae stars } \\
\text { with periods lon- } \\
\text { ger than } 0.4 \text { days }\end{array}$ & - & Weak-line stars & - & $\begin{array}{l}\text { Galactic clusters } \\
\text { containing stars } \\
\text { brighter than } \\
\text { any in the } \\
\text { Hyades }\end{array}$ \\
\hline
\end{tabular}

An important role in investigations of galactic evolution is played by research on open and globular star clusters, particularly their colour-magnitude diagrams and luminosity functions. Although the study of star clusters and associations forms the domain of another commission, we shall refer here to a few investigations which have a direct bearing on the study of the galactic population in general. Roberts [ror] has investigated whether the $\mathrm{O}$ and $\mathrm{B}$ stars form only in clusters and associations. It appears that the observed number of such stars in the Galaxy and their probable lifetimes are compatible with this assumption. Salpeter [102] has introduced the concept of an Initial Luminosity Function. This represents the distribution of the main-sequence absolute magnitudes of the stars originating from the interstellar matter per volume of space in the solar neighbourhood. It is the luminosity function which would be observed if all stars had kept their main-sequence luminosity instead of evolving towards the super-giant and later stages, and if no stars were in the process of formation any more.

The observed luminosity function deviates from this Initial Luminosity Function mainly because of the low lifetimes of the stars of high luminosity. By taking into account the most probable values of the main-sequence lifetimes of these stars, Salpeter was able to reduce the observed luminosity law to the Initial Luminosity Function, assuming a uniform rate of formation of stars in the solar neighbourhood. It was found that the total number of stars which must have moved off the main sequence is comparable with the total number of white dwarfs. Sandage [ro3], in testing the Salpeter Initial Luminosity Function, found this to agree reasonably well with the bright end of the luminosity 


\section{COMMISSION 33}

function of young open clusters, where evolutionary effects are supposed to be small. This author also showed that the luminosity function of the $\mathrm{K} \mathrm{O}-\mathrm{K} 2$ stars may be explained as the result of $(a)$ the evolution of stars from the main sequence according to the evolutionary tracks observed in open clusters, and $(b)$ the distribution of the original main-sequence absolute magnitudes as given by the Initial Luminosity Function. Another determination of the initial luminosity function of clusters was given by Van den Bergh [104].

\section{PROPOSALS AND DESIDERATA}

(I) A proposal for co-ordination of investigations of five Milky Way areas has been made by Parenago [ro5]. Meanwhile, spectral classification in these areas has been undertaken at Abastumani and Moscow, and photographic photometry at the observatories at Abastumani and Goloseevo (near Kiev). Dr P. P. Parenago proposes to discuss extension of the present participation in this project.

(2) Dr P. P. Parenago proposes to discuss the nomenclature used in indicating spiral features in the Galaxy, and to adopt the system used in the Dutch radio-astronomical papers.

(3) Revision of the galactic pole. A proposal for a revision of the galactic pole will be presented at the Moscow meeting on behalf of Sub-Commission 33 $b$, formed for this purpose at the Dublin meeting. The exact formulation of this proposal will be communicated in advance of the Moscow assembly.

President of the Commission

\section{REFERENCES}

[I] Priester, W. Z. Ap $\left\{\mathbf{3}^{8}, 73,1955\right.$.

[2] Hagen, J. P., Lilley, A. E. and McClain, E. F. Ap. J. 122, 36r, I955.

[3] Smith, F. G., O'Brien, P. A. and Baldwin, J. E. M.N.R.A.S. ז16, 282, 1956.

[4] Westerhout, G. B.A.N. 13, 105, 1956.

[5] van Woerden, H., Rougoor, W. and Oort, J. H. C.R. 244, I691, 1957.

[6] Dufay, J., Bigay, J. H. and Berthier, P. Vistas in Astronomy, I539, I956 (Pergamon Press, London).

[7] Luyten, W. J. and Carpenter, E. F. Astr. J. 60, 429, 1955.

[8] Luyten, W. J. and Seyfert, C. K. Astr. J. 6r, 264, 1956.

[9] Iriarte, B. and Chavira, E. Boletin Tonantzintla y Tacubaya, no. 16, 1957.

[10] Shapley, H. Vistas in Astronomy, I564, 1956.

[II] Schmidt, M. B.A.N. 13, 247, 1957.

[12] Kerr, F. J., Hindman, J. V. and Carpenter, M. S. Nature, 180, 677, 1957.

[13] Kerr, F. J. and Hindman, J. V. P.A.S.P. 70, $195^{8}$.

[14] Edmondson, F. K. P.A.S.P. 67, to, 1955 .

[15] Westerhout, G. B.A.N. 13, 201, I957.

[16] Lilley, A. E. Ap.J. I2x, 559, I955.

[17] Heeschen, D. S. $A p . J$. I21, 569, 1955.

[18] Davis, R. J. Ap. J. I25, 39I, 1955.

[19] Helfer, H. L. and Tatel, H. E. Ap. J. I21, 585, 1955.

[20] Parenago, P. P. Astr. J. Moscow, 32, no. 3, I955.

[2I] Morgan, W. W., Code, A. D. and Whitford, A. E. Ap. J. Suppl. 2, 4I, 1955.

[22] Hiltner, W. A. Ap. J. Suppl. 2, 389, 1955; Contr. McDonald Obs. no. 269.

[23] Hoffleit, D. $A p . J .124,6 \mathrm{I}, 1956$.

[24] Münch, G. $A p . J$. 125, 42, 1957.

[25] Kurochkin, N. E. Astr. J. Moscow, 34, no. I, 1957.

[26] Kiang, T. Ann. Astrophys. 18, 76, 1955.

[27] Stibbs, D. W. N. M.N.R.A.S. II5, 363, 1953

[28] Feast, M. W., Thackeray, A. D. and Wesselink, A. J. Mem. R.A.S. 67, 5I, I955 and 68, I, 1957 . 


\section{STRUCTURE DU SYSTEME GALACTIQUE}

[29] Stibbs, D. W. N. M.N.R.A.S. 116, 453, 1956.

[3०] Gascoigne, S. C. B. and Eggen, O. J. M.N.R.A.S. x17, 430, 1957.

[31] Petrie, R. M., Cuttle, P. M. and Andrews, D. H. Astr. J. 6r, 289, 1956.

[32] Weaver, H. F. Astr. J. 60, 202 and 208, I956.

[33] Mohr, J. M., Mayer, P. and Stohl, J. Bull. Astr. Insts. Czech. 8, 5, 1956.

[34] Pskovsky, J. P. Astr. J. Moscow, 34, no. I, 1957.

[35] de Vaucouleurs, A. M.N.R.A.S. 117, 449, 1957.

[36] Bidelman, W. P. P.A.S.P. 69, 147 and 326, 1957 .

[37] Halliday, I. $A p . J$. 122, 222, 1955.

[38] Roman, N. G. Ap. J. Suppl. 2, 198, 1955; Contr. McDonald Obs. no. 262.

[39] Sharpless, S. Ap. J. r24, 342, 1956.

[40] Chalonge, D. Ann. Astrophys. 19, 258, 1956; Vistas in Astronomy, 1328, 1956.

[4I] Strömgren, B. and Gyldenkerne, K. Ap. J. 12I, 43, I955; Gyldenkerne, K. Ap. J. 121, 38, 1955; Naur, P. Ap. J. 122, 182, 1955; Strömgren, B. Vistas in Astronomy, I336, 1956.

[42] Petrie, R. M. Vistas in Astronomy, 1346, 1956; Petrie, R. M. and Moyls, B. N. Publ. Dom. Astrophys. Obs. Victoria, Io, no. I3, 1956.

[43] Stock, J. Ap. J. 123, 253, I956.

[44] Wilson, O. C. and Vainu Bappu, M. K. Ap. J. 125, 661, 1957.

[45] Gliese, W. Mitt. Astr. Recheninst., Heidelberg, series A, no. 8, 1957.

[46] Gliese, W. Z. Ap. 39, I, 1956.

[47] Eggen, O. J. Astr. J. 62, 45, I957; Lick Obs. Bull. no. 549.

[48] Johnson, H. L. and Knuckles, C. F. Ap. J. 126, II3, 1957.

[49] Kron, G. E., Gascoigne, S. C. B. and White, H. S. Astr. J. 62, 205, 1957; Lick Obs. Bull. no. 556 .

[50] Mumford, G. S., III. Astr. J. 6r, 224, 1956; Publ. McCormick Obs. II, part II.

[5I] Dyer, E. R., Jr. Astr. J. 6r, 228, 1956; Publ. McCormick Obs. II, part 12.

[52] Wehlau, A. W. Astr. J. 62, 169, 1957.

[53] Einasto, J. Tartu Pub. 33, 35 and 57, 1955.

[54] Nahon, F. C.R. 242, 462, 1956; 244, 2775 and 2688, 1957.

[55] Ewart, D. G. M.N.R.A.S. II5, 47, 1955.

[56] Vyssotsky, A. N. P.A.S.P. 69, 109, I957; Publ. McCormick Obs. 12, part 4.

[57] Edmondson, F. K. Astr. J. 6r, 175, I956.

[58] Malmquist, K. G., Schalén, C. and Westerlund, B. Ann. Uppsala Obs. 4, no. I, 1954

[59] Ramberg, J. M. Ann. Stockholms Obs. 20, no. I, 1957.

[6o] Bok, B. J. Astr. J. 61, 309, 1956.

[61] Bok, B. J. Vistas in Astronamy, I522, 1956.

[62] Balz, A. G. A., Jr. Publ. McCormick Obs. 13, part I, 1956.

[63] Nassau, J. J., Blanco, V. M. and Cameron, D. M. Ap. J. 124, 522, 1956.

[64] Blanco, V. M. and Nassau, J. J. Ap. J. r25, 408, 1957.

[65] Smith, E. and H. J. Astr. J. 6r, 273, 1956.

[66] Nassau, J. J. and Blanco, V. M. Ap. J. 125, I95, 1957.

[67] Sanduleak, N. Astr. J. 62, I50, 1957.

[68] Velghe, A. G. Ap.J. 125, 728, 1957.

[69] van Rhijn, P. J. Publ. Kapteyn Lab. Groningen, no. 57, 1955 and no. 59, 1957.

[70] Nahon, F. Bull. Astr. Paris, 21, 55 and 93, 1957.

[7I] Woolley, R. v. d. R. M.N.R.A.S. 117, 198, 1957.

[72] Kuzmin, G. Tartu Pub. 33, 3, 1955.

[73] Eelsalu, H. Tartu Pub. 33, I 53, r958.

[74] Panaiotov, L. A. Astr. Circ. U.S.S.R. no. I71, 1956.

[75] McCuskey, S. W. Ap. J. 123, 458, 1956.

[76] Morgan, H. R. Astr. J. 6r, 90, 1956.

[77] Filin, A. J. Astr. J. Moscow, 34, no. 4, 1957.

[78] Blaauw, A. $A p . J$. 123, 408, 1956.

[79] Daube, I. A. Astr. J. Moscow, 32, no. 4, 1955. 


\section{COMMISSION 33}

[80] Blaauw, A. P.A.S.P. 68, 495, 1956.

[8I] Safronov, V. S. Bull. Var. Stars, 10, 236, 1955.

[82] Joy, A. H. P.A.S.P. 67, 420, 1955 .

[83] Oosterhoff, P. Th. B.A.N. 13, 67, 1956.

[84] See: Mayall, N. U. and Bidelman, W. P. P.A.S.P. 67, 335, 1955.

[85] Shazova, R. B. Astr. J. Moscow, 33, no. 6, 1956.

[86] Pavlovskaja, E. D. Astr. J. Moscow, 33, no. 5, 1956.

[87] Heintz, W. D. A.N. 282, 22 I, 1955.

[88] Kurth, R. Introduction to the Mechanics of Stellar Systems (Pergamon Press, London, 1957).

[89] Camm, G. L. Vistas in Astronomy, 216, 1955.

[9o] Schmidt, M. B.A.N. 13, I5, 1956.

[91] Kuzmin, G. Astr. J. Moscow, 33, 27, 1956; Tartu Teated, no. 2.

[92] Kuzmin, G. Tartu Teated, no. 3, 1956.

[93] Contopoulos, G. Ap.J. 124, 643, 1956.

[94] Contopoulos, G. Ann. Stockholms Obs. 19, no. 10, I957; 20, no. 5, 1958.

[95] Mikisha, A. M. and Tsitsin, F. A. Astr. J. Moscow, 34, no. I, 1957.

[96] van Wijk, U. Astr. J. 61, 277, 1956.

[97] Idlis, G. M. Astr. J. Moscow, 33, no. r, 1956; Bull. Alma-Ata Astrophys. Inst. 4, 1957.

[98] Perek, L. A.N. 283, 213, 1956; Veröff. Astr. Inst. Brno, no. 2.

[99] Lindblad, B. Ann. Stockholms Obs. 18, no. 6, 1955; 19, no. 7, 1956 and no. 9, 1957.

[100] Morgan, W. W. and Mayall, N. U. P.A.S.P. 69, 291, 1957.

[101] Roberts, M. S. P.A.S.P. 69, 59, 1957.

[102] Salpeter, E. E. $A p . J .121,161,1955$.

[103] Sandage, A. Ap. J. 125, 422, 1957.

[104] van den Bergh, S. $A p . J$. 125, 445, 1957.

[105] Parenago, P. P. Astr. J. Moscow, 33, no. 5, 1956.

\section{3a. SUB-COMMISSION ON THE CO-ORDINATION OF GALACTIC RESEARCH}

The following partial summary of the proceedings of the Symposium on 'Co-ordination of Galactic Research', held in Stockholm in June 1957, may serve as a report of the Sub-Commission.

\section{Galactic clusters and $O$ associations}

It has become more and more evident how extremely important the study of galactic clusters and $\mathrm{O}$ associations is for the investigation of galactic structure. This is so for three reasons:

(I) For locating spiral structure independent of the velocity. It is difficult to stress this point sufficiently. We can hope to determine the scale of the Galactic System from them, as well as spiral structure.

(2) Most important of all: they can give information on systematic deviations from circular motion.

(3) They lend themselves excellently for a determination of age, for a determination of change of stellar composition with time and for the derivation of semi-empirical paths of evolution.

The study of clusters may eventually teach us something about differences in distribution and motions of objects formed at various epochs in the development of the Galactic System.

What we need is, first, to discover more distant clusters, secondly, to find older clusters, thirdly, to obtain colour-magnitude diagrams of good accuracy for all clusters (this involves the problem of identifying members), fourthly, radial velocities. In connexion with the latter item I want to refer to the report by Weaver on the valuable material 


\section{STRUCTURE DU SYSTEME GALACTIQUE}

on radial velocities of stars in galactic clusters obtained by Trumpler. Weaver has reported that these observations are now being prepared for publication.

At the instigation of Heckmann and Haffner the feasibility of obtaining, in a number of regions around the sky, standard magnitudes and colours to be used for calibrating the photometric data for individual clusters was investigated during the meeting by a small committee. A positive proposal worked out by this committee was presented by Walker.

\section{Cepheids}

Kukarkin has stressed again the desirability of searches for more distant $\delta$ Cephei variables. There can be no doubt that this offers one of the most important ways of investigating galactic structure and dynamics. This has been borne out again by the report given by Oosterhoff on the colour-excesses of southern cepheids and the space distribution derived with the aid of these colours.

The amount of work involved in new surveys will be very large, and co-ordination is therefore important. It is evidently essential that accurate colour-excesses and radial velocities be determined for all $\delta$ Cephei variables that will be found.

Kulikovsky has proposed that more extensive investigations be made of trapeziumtype groups, as well as of wide double and multiple stars in general.

In connexion with the general topic of this conference mention should be made of the fact that a co-ordinating commission for $T$ associations was formed during the Burakan symposium on Non-stable Stars; members are Haro, Herbig and Kholopov.

\section{Spiral structure}

Since the Groningen conference a great mass of new information on the distribution of neutral hydrogen has become available. The observations concerning the southern parts of the Milky Way are now being reduced. A new development which appears particularly intriguing is the discovery of a spiral arm in the nuclear region which moves away from the centre at a velocity of $53 \mathrm{~km} / \mathrm{sec}$. Other structures, closer to the centre, show still larger deviations from circular motion.

The report of the Groningen conference stressed the importance of studying possible differences between the distributions of A stars, $\mathrm{K}$ giants and the neutral hydrogen. Ramberg has reported on his work on $A$ stars and $K$ giants. Though indicating some relation with the nearby spiral structure of the gas the investigations do not yet provide sufficiently detailed information and should be extended to larger distances and to more regions before they can give a final answer to the question.

\section{Special regions}

Parenago has made a proposal to study a number of special regions (see p. 520). Undoubtedly it is of considerable value to concentrate efforts on a few special regions, and those selected are undoubtedly important ones. It was therefore proposed that the conference agrees in principle to the plan and advocates that support be given to it as far as possible. It was stressed at the same time that attention should be concentrated on individual data of very great accuracy rather than on rough data for great numbers of stars. It is still uncertain how much value the determination of proper motions will have in connexion with the study of the space structure of these regions.

\section{Accurate spectrophotometry}

The possibility of accurate spectrophotometric determinations of spectral types, luminosities and possibly a third parameter has been discussed by Chalonge, Strömgren and Miss Roman. The subject had been much discussed at our previous symposium; since then a large amount of data has been collected and the great advances that may be obtained through this type of accurate measurement have been amply demonstrated. Here indeed is an enormously rich mine for observational programmes of the greatest importance for galactic research. 


\section{COMMISSION 33}

Miss Roman's investigations of high-velocity stars have indicated that the ultra-violet excess is well correlated with average velocity, and may therefore be an important indicator of age for the types of stars she studied.

$\mathrm{O}$. C. Wilson has recently shown that the width of the emission core of the $\mathrm{K}$ line in $\mathrm{G}, \mathrm{K}$ and $\mathrm{M}$ stars is strongly correlated with absolute magnitude, while being independent of spectral type. This might prove to be the most accurate method for determining absolute magnitudes. But high dispersions are required. Co-ordination with other accurate measures of luminosity is important.

It was proposed that parallax observers would be asked to determine accurate trigonometric parallaxes of about two dozen stars with very weak lines.

\section{Determination of $K_{z}$}

This still leaves much to be desired. The best prospects would seem to be offered by accurate objective-prism classifications of $\mathrm{K}$ stars down to about $14^{\mathrm{m}} \mathrm{O}$ in the galactic polar caps, preferably in Kapteyn's Selected Areas where photometric and propermotion data are already available. It would be desirable to make measurements that would separate giants from sub-giants and dwarfs. Knowledge of radial velocities down to the same magnitude limit would be of the greatest importance.

\section{Radial velocities}

Progress with the objective-prism method, as reported by Fehrenbach, is extremely promising. Similar work will be taken up at the Royal Greenwich Observatory. Co-operation for obtaining a sufficient number of faint standard velocities is desirable.

\section{Nucleus and disk}

The desirability of paying more attention to long-period variables for investigating the older disk population was stressed by Kukarkin and strongly supported by the meeting.

\section{Halo}

The search for RR Lyrae variables in a number of regions in moderate galactic latitudes which was advocated at the Groningen conference has meanwhile been undertaken, and has been reported at this meeting. Complete sets of plates down to a faint magnitude limit have been obtained with the 48-inch Schmidt on Mount Palomar and are now being searched for variables by Plaut in Groningen. An essential desideratum still to be provided is colour excesses for all the variables found.

Morgan has reported on the striking difference in the spectra of various globular clusters. These indicate that there is a class of clusters which is relatively rich in heavy elements and which is probably concentrated towards the galactic disk. However, there are likewise considerable differences in composition among clusters having large distances from the galactic plane.

Further investigations of spectra, as well as colours and magnitudes of individual stars in globular clusters are highly desirable.

\section{Magellanic Clouds}

Thackeray reported on some of the work on the Magellanic Clouds that is now being done, or that would seem of importance to be done in the nearest future. This latter comprises objective-prism surveys to $13^{\mathrm{m}} \cdot 5$, objective-prism radial velocities to eliminate foreground objects, a search for bright variables, further photometry of known variables and a general search for new ones, in particular also for those of long period. An atlas of charts should be made, covering a larger field than the Harvard charts.

$$
\begin{gathered}
\text { J. H. OORT } \\
\text { President of the Sub-Commission }
\end{gathered}
$$




\section{3b. SUB-COMMISSION FOR THE DETERMINATION OF THE GALACTIC POLE AND OF GALACTIC LONGITUDES}

This Sub-Commission was appointed at the ninth General Assembly in Dublin 'to investigate the desirability of a revision of the galactic pole and of the zero point of galactic longitude'. A circular letter reporting its conclusions and recommendations was distributed among the members of Commissions 33 and 40 and other interested persons about a month before the meetings, as a basis for the discussions in a joint meeting of Commissions $33,33 b$ and 40 . The full text of the five items discussed in this circular letter is reproduced in the following paragraphs. For the adopted resolution, we refer to the Report of the joint meeting.

A report containing details of the radio data relevant to the choice of a galactic coordinate system was circulated to astronomers interested in the subject in June I958 (Gum and Pawsey, I958) [r]. Some copies of a report on the determination of the galactic pole from $21 \mathrm{~cm}$ line data were distributed in Moscow (Gum and Kerr, I958) [2]. Both reports, as well as other articles bearing on the problem of the new galactic co-ordinate system, will be published in one of the leading astronomical journals during I959.

\section{CONTENTS OF CIRCULAR LETTER}

\section{(I) Terms of reference}

The Sub-Commission was appointed at the Dublin General Assembly of the I.A.U. in I955 'to investigate the desirability of a revision of the galactic pole and of the zero-point of galactic longitude'.

\section{(2) Scope of report}

The Sub-Commission has examined the basis for a possible revision of the galactic pole, taking into account all observational radio and optical data available at the time of writing of this report.

It recommends the adoption in the near future of a standard system of galactic coordinates, to be used as a reference system both for radio and optical investigations and primarily based on:

(a) The distribution of neutral hydrogen in the inner regions of the Galaxy.

(b) The position of the radio source Sgr A, which is with very little uncertainty identified with the galactic nucleus.

The exact co-ordinates of the galactic pole and the galactic centre to be adopted will be shortly available to the Sub-Commission, pending the final reduction of already available observations. A resolution which would give effect to these recommendations is given below.

\section{(3) Proposed resolution}

The Sub-Commission proposes the following resolution to be put before the General Assembly.

Commissions 33 and 40 recommend:

(a) The adoption of a standard system of galactic co-ordinates of which the pole is based primarily on the distribution of neutral hydrogen in the inner parts of the galactic system.

(b) (i) That the zero of longitudes be the longitude of the galactic nucleus; (ii) that longitude be counted from $-\mathrm{I} 80^{\circ}$ through o to $+\mathrm{I} 80^{\circ}$, in the same direction as in the current system, and that latitude be counted in the conventional manner from $-90^{\circ}$ through o to $+90^{\circ}$.

(c) That Sub-Commission $33 b$ be authorized to define the exact values of the coordinates of the pole and of the zero of longitudes immediately after the final reduction of the relevant observations is finished. 


\section{COMMISSION 33}

(d) That Sub-Commission $33 b$ be charged with the communication of these values to the members of the I.A.U. and to all other interested institutions and individuals.

(e) That Sub-Commission $33 b$ be charged with the supervision of the publication of tables, accurate to $0: 01$, necessary for the conversion from galactic into equatorial coordinates and vice versa, and from co-ordinates based on the Lund pole into the newly defined system and vice versa, and of conversion charts.

$(f)$ That the following symbols be used for the new galactic co-ordinates:

...for the longitude, and

...for the latitude, both as defined above.

(g) That the following names be used for these new co-ordinates:

...for the longitude, and

...for the latitude.

\section{(4) Evidence on the locations of the pole and the centre of the galactic system}

(a) The Pole. Precise evidence on the location of the galactic pole depends on the study of objects strongly concentrated towards the galactic plane. The relevant observations consist of (i) radio observations at $2 \mathrm{I} \mathrm{cm}$ of neutral interstellar hydrogen ( $\mathrm{H}$ I regions), (ii) radio observations of the continuum which are now available in the range $20 \mathrm{~cm}$ to $3.5 \mathrm{~cm}$, and (iii) optical observations.

The effective optical observations are restricted to a relatvely small region around the Sun about $3000 \mathrm{pc}$ in radius, that is, to an area of 5 or $10 \%$ of that accessible to radio observations; the objects concerned represent less than $0 \cdot 1 \%$ of the total mass of the Galaxy. The $2 \mathrm{rcm}$ radio observations pertain to the neutral hydrogen which represents about $2 \%$ of the total mass of the Galaxy, and which can be located over the greater part of the galactic plane out to a radius of about $15 \mathrm{kpc}$ from the galactic centre. The radio continuum observations give only integrated effects along the line-of-sight and are most effectively used in checking a model based on other data.

The outstanding feature of the distribution of neutral hydrogen is the high degree of flatness of the layer in the region of the Galaxy within about $7 \mathrm{kpc}$ of the centre. Within this area nearly all the points of maximum density of hydrogen (smoothed over areas of about $\mathrm{I} \mathrm{kpc} \mathrm{kP}^{2}$ ) lie within $20 \mathrm{pc}$ of the mean plane of the layer (which is described as the 'H I Principal Plane'). These deviations are within I part in 400 of the diameter of the region. In the outer regions of the Galaxy systematic distortions of up to 800 pc occur. The high degree of flatness of the disk of interstellar gas in the inner region is of fundamental physical significance and the $\mathrm{H}$ I principal plane therefore appears to form the best reference plane for the galactic co-ordinate system. It thus seems reasonable to choose the galactic plane, that is, the equatorial plane of the galactic co-ordinate system, as passing through the Sun and parallel to the $\mathrm{H}$ I principal plane. Available observations locate the corresponding galactic pole about $I^{\circ} 6$ from the current pole with an uncertainty of about $0^{\circ} \mathrm{I}$. Provisional position $l \approx 350^{\circ}, b \approx+90^{\circ}-\mathrm{I}^{\circ} 6$.

Optical observations of different groups of objects give poles which are scattered over a range of several degrees and an optical determination of the pole depends on the choice of the group, or groups, of objects to be given the most weight. We have thought it proper to give the greatest weight to observations of the more distant objects (for example, cepheids, OB-stars, and open clusters at distances of about $3000 \mathrm{pc}$ ). This weighting corresponds to the assumption that, in the vicinity of the Sun, local irregularities and effects of selection are more serious than possible large-scale distortions. It is supported by the greater consistency of the results from the more distant objects. These selected optical data give consistent agreement with the $\mathrm{H}$ I pole within about $0^{\circ} \cdot 2$ for different optical groups.

In the radio continuum results the important feature for our purposes is that, in the range of directions within about $\pm 50^{\circ}$ of the galactic centre, where the $\mathrm{H} \mathrm{r}$ layer is flat, the 


\section{STRUCTURE DU SYSTEME GALACTIQUE}

'ridge lines' of the continuum contour maps are very well defined and agree closely with one another and with the position of the $\mathrm{H}$ I layer. In directions where the $\mathrm{H}$ I layer is distorted the continuum ridge line is ill defined and appears to conform with the $\mathrm{H} \mathrm{I}$ irregularities. The inference is that, on a large scale, the sources of the 'disk component' of the continuum are distributed similarly to the $\mathrm{H}$ I regions. The agreement is within the observational uncertainty of about $o^{\circ} \mathrm{x}$.

(b) The height of the Sun above the plane. Van Tulder's optical studies show that the Sun is about Io or I5 pc north of the mean plane of extreme population I objects in the vicinity of the Sun. But it is not yet possible to say if these local population i objects are in the $\mathrm{H}$ I principal plane or are systematically above or below it by a distance of this order. Preliminary analyses indicate that the Sun is very near to the $\mathrm{H}$ I principal plane. The distance $z_{0}$ of the Sun from this plane does not appear explicitly in the parameters of the co-ordinate system, as this system is necessarily based on a galactic plane through the Sun. It is involved in determinations of the plane of symmetry of various groups of objects in the Galaxy, such as the sources of the continuous radio emission.

(c) The longitude of the galactic centre. The longitude of the galactic centre derived from various radio and optical studies is $l=327^{\circ} 5 \pm I^{\circ}$. The provisional position of the radio source Sgr A ( $\left.{ }_{7} \mathrm{~S} 2 \mathrm{~A}\right)$ is $l=327^{\circ} 70 \pm 0^{\circ} 05, b=-\mathrm{I}^{\circ} \cdot 46 \pm 0^{\circ}{ }^{\circ} 05$, which agrees within one-fifth of the observational uncertainty with the position just mentioned. The source lies within the limits of uncertainty in the $\mathrm{H}$ I principal plane. We shall assume that Sgr A is identical with the galactic nucleus, which appears highly probable.

\section{(5) Considerations relevant to the setting up of a new system of co-ordinates}

(a) Should a new system be set up now? The current system of galactic co-ordinates does not serve its purpose properly because its pole is displaced from the pole of the $\mathrm{H} \mathrm{I}$ principal plane and from that of population $\mathrm{I}$ objects near the Sun by about $x^{\circ} \div 6$. Further the zero of longitude is in an inconvenient position, about $32: 5$ from the direction of the galactic centre.

The new system to be adopted may not be perfect for future refined studies: yet we recommend a new system now on the following grounds:

(i) The current pole is sufficiently seriously misplaced to make the discrepancy obvious for a large proportion of the observations. Hence observers are likely to use their own systems if the I.A.U. does not recommend a new one.

(ii) The anticipated improvement, by a factor of ten-to-one or better, is substantial, and is unlikely to be much increased for many years.

(iii) Further, a co-ordinate system is placed with adequate accuracy when the scatter of the objects or groups of objects considered is considerably greater than the uncertainty in the position of the plane due to observational errors, or due to the choice of the objects from which the plane is determined. The proposed system appears to satisfy this condition for the great majority of both optical and radio objects.

(iv) This is a time in the history of astronomy when a great increase in galactic studies involving the use of galactic co-ordinates is anticipated. Consequently the introduction of a new system now, rather than in a few years, will prevent a considerable amount of conversion between the conventional and the new co-ordinate systems.

(b) The type of system. We recommend a latitude and longitude system basically similar to the current one, with two modifications. We propose (i) to place the zero of longitude in the direction of the longitude of the galactic centre, and (ii) to count longitudes from $-180^{\circ}$ through $0^{\circ}$ to $+180^{\circ}$ in the same direction as in the current system.

We consider these changes desirable now that the Sun is known to be well removed from the galactic centre and the direction of the centre is well established. With our present knowledge, the Galaxy, viewed from the Sun, has two planes of pseudo-symmetry: the Galactic plane and the plane normal to this passing through the Sun and the galactic centre. The justification for the use of positive and negative longitudes is the ease of recognition of corresponding longitudes equally displaced from the zero of longitudes. 


\title{
COMMISSION 33
}

The galactic equator is defined by a plane through the Sun which will not coincide with the $\mathrm{H}$ I principal plane if the Sun is at a finite distance from it. Accordingly the position of the galactic centre, if this is situated in the $\mathrm{H}$ I principal plane, will not be at latitude zero, and the latitude of points in the H I principal plane will be a function of their distance from the Sun.

\author{
A. BLA A UW \\ J. L. PAWSEY \\ G. WESTERHOUT
}

REFERENCES

[I] Gum, C. S. and Pawsey, J. L. Radiophysics Laboratory Report, no. 137, Sydney, June I958. [2] Gum, C. S. and Kerr, F. J. Radiophysics Laboratory Report, no. r38, Sydney, July I958.

President: A. Blaauw.

$$
\text { Report of Meeting. I5 August } 1958
$$

SeCRetary: L. Perek.

The Draft Report was adopted after a few changes had been proposed and accepted.

The President reported on the main conclusions of the symposium on Co-ordination of Galactic Research (I.A.U. Symposium, no. 7) held at Stockholm in I957 (see also the report of Sub-Commission 33a).

Upon invitation by the President, Dr B. Strömgren and Miss N. G. Roman summarized their recent work on the photometric separation of population groups. It was pointed out by the President that this work has an important bearing on future planning of galactic research. Partial descriptions of the work by Dr B. Strömgren may be found in his Halley lecture I958 and in his paper presented at the symposium on the HertzsprungRussell Diagram (I.A.U. Symposium, no. Io).

Miss N. G. Roman reported on a correlation between the height of a star above the normal $U-B$ vs $B-V$ curve, which is called the ultra-violet excess, and its velocity in space. If the stars in the high-velocity catalogue by the author are grouped by ultraviolet excess, the mean galactic rotation velocity decreases, and the velocity dispersion in each of three cartesian co-ordinates increases, as the ultra-violet excess increases. In view of this correlation, the ultra-violet excess appears to be a useful criterion for separating population groups. As a test, photo-electric observations on the $U-B-V$ system were obtained for about rooo stars. Of the stars reduced so far one group with ultra-violet excesses of $0.15^{\mathrm{m}}$ or larger was compared with another group with small excesses. Stars with tangential velocities larger than $60 \mathrm{~km} / \mathrm{sec}$ represented $55 \%$ of the whole list, and the group with large excesses $20 \%$. All of the stars with low excesses are within $500 \mathrm{pc}$ of the galactic plane but only $55 \%$ of the stars in the other group are within this limit.

The proposal by Prof. P. P. Parenago, to adopt formally the names Orion arm and Perseus arm for some of the spiral features was discussed and the decision was postponed to a next meeting of the Union when more complete information on the $\mathrm{H}$ I distribution will be available.

Acting upon a request from the Director of the Cordoba Observatory, Dr L. Gratton, the President inquired whether the Commission would be inclined to support an application by Dr Gratton at the Finance Committee for a grant to cover the cost of photoprinting the Cordoba Durchmusterung. A grant of about 3000 U.S. dollars would be sufficient for 150 copies, which would then be sent free of charge to all institutions asking for them. The Commission was of the opinion that there certainly is a need for making the Cordoba Durchmusterung available again, but that the number of institutions interested should be more accurately estimated by means of a circular letter to all observatories.

The President asked the audience to be prepared for a reduction of the membership of the Commission in accordance with the policy of the Executive Committee and with the statement made in the introduction of the Draft Reports. 


\section{STRUCTURE DU SYSTEME GALACTIQUE}

B. J. Bok proposed that a Conference on Co-ordination of Galactic Research be held in I96o in Australia. The travel expenses from Europe to Australia are admittedly high, but on the other hand it is advisable to have such conferences also outside the European area where they have been held so far.

Miss N. G. Roman moved that the proposal no. I4 $(h)$ of the agenda of the General Assembly 'that Commission 32 on Selected Areas be discontinued, but that a new SubCommission on Selected Areas be established as Sub-Commission 33c' have the support of the members of Commission 33. The motion was seconded by B. J. Bok and carried (see Resolution no. 60).

\section{Report of Joint Meeting of Commissions 27 and 33. I8 August 1958}

President: A. Blaauw.

Secretary: L. Perek.

The President drew attention to the new edition of the General Catalogue of Variable Stars and conveyed the congratulations of the members of the Commissions to the authors of this excellent work.

Dr C. Hoffmeister reported about work on variable stars at the Sonneberg Observatory. Several members of the staff and guests are investigating variables brighter than $\mathrm{I} 2^{\mathrm{m}}$, main sequence variables, and long-period variables. The current investigations of Milky Way fields are nearly complete with the exception of part of the short-period variables. The counts, necessary for evaluating the numbers of variables taking into account the probability of the discoveries, have been made.

Among 79 new variables found in the area 73 Her, 56 were RR Lyrae stars. A search for faint RR Lyrae stars near the galactic pole with a limiting magnitude $I 8$ revealed in four fields of I4 square degrees each only one RR Lyrae star of about $I 7^{\mathrm{m}}$. This would seem to indicate a rather flat shape of the galactic halo. Dr W. Luyten remarked that he found faint blue stars down to $22^{\mathrm{m}}$ and that thus the halo must extend to large distances from the galactic plane. The work at Leiden was summarized by Prof. P. Th. Oosterhoff. Photo-electric measures were made in two colours of about seventy stars in SA I82 between 8.5 and $15.5 \mathrm{SPg}$ magnitude. There are now four Franklin-Adams fields where all variables have been measured. The limiting magnitude is 16.5 . It is intended to attain an accuracy of $\pm 0 \cdot 2^{\mathrm{m}}$ for the system of reference stars; however, it is not yet certain that this will be feasible.

J. Ponsen, of Leiden, recently investigated two small fields of 0.27 square degrees in Sagittarius on Ioo plates taken at the Radcliffe Observatory. The limiting magnitude was about I9.5 photographic. By comparing 20 pairs of plates, Io4 and 64 variables have been found in the fields Sgr I and Sgr II, respectively. In both fields the frequency of the periods of the RR Lyrae stars shows a well-defined minimum around $0 \cdot 4$ day. The great majority of the periods fall on the longer side of this minimum. This minimum does not represent the separation between $a$ - and $c$-type light-curves. The RR Lyrae stars in Sgr I are on the average one magnitude fainter than those in Sgr II. This is caused by interstellar absorption which is heavier and less uniform in Sgr I than in Sgr II. Two cepheids have been detected in Sgr I.

In reply to a question by Dr P. N. Kholopov, Oosterhoff stated that he was quite sure that only very few periods were spurious since the plates have been taken in a wide range of hour-angles.

Mrs M. W. Mayall reported on the activities of the A.A.V.S.O. About I5o active observers have on their lists 800 stars and make 60,000 observations a year. In the near future six photo-electric photometers will be put into operation.

The President read the report by Dr D. H. Menzel on variable-star projects at Harvard College Observatory. Dr S. Gaposchkin studied 223 variables in the Andromeda Nebula and is engaged in a search for new variables in the globular clusters $\omega$ Centauri and 


\section{COMMISSION 33}

47 Tucanae. It is planned to extend the work of the 'Milton Bureau' to fainter variables. M. Olmsted has finished standard sequences of stellar magnitudes in red and blue in seven southern fields for use in the determination of photographic colour indices of cepheids. The VSF 235 is being investigated by Dr H. Shapley and J. Kloss. A. Young is collecting data on classical cepheids in order to relate their distribution and motion to galactic structure.

Dr M. F. Walker drew attention to the star RW Trianguli. Its light-curve is similar to those of UX UMa and DQ Her in that there are shoulders before and after eclipse, an asymmetric rising branch of the eclipse-curve, a depression of the light-curve at $0.7 P$, and no detectable secondary minimum. Further the brightness outside eclipse, as well as the depth of eclipse, are variable and no reliable elements of the system can be obtained. Further observations of this star are planned for the fall of 1958 .

Dr K. A. Grigorievski reported on polarimetric observations of $\mu$ Cephei. A correlation between the degree and the angle of polarization has been detected.

Dr R. H. Stoy spoke on his proposal to prepare a catalogue of stellar magnitudes consisting of three sections. The first section will contain several hundred stars with external probable errors not exceeding $\mathrm{o}^{\mathrm{m}} \mathrm{or}$. In the second section several thousand stars will be listed with probable errors up to $\mathrm{o}^{\mathrm{m}} \mathrm{O2}$. Precepts for reducing various systems to that of the catalogue will form the third section. In reply to a question by M. F. Walker whether this is an attempt to create a new system, Stoy said that the catalogue will be a critical compilation of existing material and will be based on a rationalised $U-B-V$ system. The President read the text of the proposed recommendation and the motion was seconded and carried (see resolution no. 42).

At Cordoba Observatory a detailed study of the velocity curve during one beat-period of AI Velorum is in progress. The region of $\eta$ Carinae is being studied and faint variables have been searched for. All long-period variables south of $-25^{\circ}$ down to roth photographic magnitude at maximum are being observed.

\section{Report of Joint Meeting of Commissions 33, 33b and 40. I9 August I958}

President: A. Blaauw.

SECRETARY: G. Westerhout.

The originally scheduled joint meetings of Commissions $33,33 b$ and 40 and of Commissions 33 and 40 were combined into one three-hour session, to discuss the report by SubCommission $33 b$ on the revision of the galactic co-ordinate system, and the resolution concerning this revision for adoption by the General Assembly.

The President reviewed the problem and enlarged on various points of the report of the Sub-Commission. Dr G. Westerhout presented the evidence on the position of the galactic plane as obtained from $2 \mathrm{I}-\mathrm{cm}$ line observations of the distribution of neutral hydrogen. Dr J. L. Pawsey presented data on the angular distribution of the continuous radiation at radio wave-lengths. The President discussed optical data bearing on the problem. In the discussion following it was agreed that the optical and radio continuum data should be used only for the purpose of checking the results from the neutral hydrogen observations. Item $(a)$ of the proposed resolution was then carried by the members of Commissions 33 and 40 .

The arguments in favour of choosing the longitude of the galactic centre as the zero of galactic longitudes were given by Blaauw, Westerhout and Oort, who presented data on the optical position, the radio continuum measurements, the position of the radio source Sgr A and the reasons why this source is believed to be the nucleus of the galactic system. The latter point in particular was treated in some detail by Prof. J. H. Oort, who discussed the Dutch observations of the expansion of neutral hydrogen gas away from the galactic centre. In the subsequent discussion, the need for position measurements of Sgr A at more than one frequency was stressed. It was made clear that once the best 


\section{STRUCTURE DU SYSTEME GALACTIQUE}

possible position for the zero of longitudes has been chosen by Sub-Commission $33 b$, this position should remain fixed regardless of later improvements of the position of Sgr A. The present data suggest that such improvements will be of the order of a few hundredths of a degree at most. The question as to whether the zero of longitudes should be near the position of the galactic nucleus was discussed at length. Points in favour are its use in theoretical work, its now sufficiently well-known position, and the smaller chance of confusion between old and new co-ordinates. Points against are the need for one more step in the conversion from equatorial into galactic co-ordinates, and the inconvenience for those who are used to the old system.

The President summarized arguments for and against the counting of longitude from $-180^{\circ}$ through $0^{\circ}$ to $+180^{\circ}$. The proposal evoked considerable discussion. Serious arguments against it are the addition of one more sign, and the difficulty of distinguishing between longitude and latitude without adding the symbols. The meeting then resolved to recommend the following wording for item $(b)$ of the proposed resolution:

(b) (i) That the zero of longitudes be chosen near the longitude of the galactic nucleus, (ii) that longitude be counted from $0^{\circ}$ to $360^{\circ}$ in the same direction as in the current system, and that latitude be counted in the conventional manner from $-90^{\circ}$ through $0^{\circ}$ to $+90^{\circ}$.

Items $(c),(d)$ and $(e)$ of the proposed resolution were carried after some discussion on the question whether final values of the co-ordinates could not be adopted during the present session of the I.A.U. The meeting further agreed that the members of SubCommission $33 b$, perhaps after consultation with various authorities, should decide on the epoch of the tables mentioned in $(e)$.

Various suggestions were made for new symbols for the new galactic co-ordinates, and for a name for the new system. The meeting finally agreed on the following wording of items $(f)$ and $(g)$ :

(f) That the new galactic co-ordinates be designated by the same symbols as the old galactic co-ordinates.

(g) That the revised system be referred to as the galactic co-ordinates (I958 revision).

It was decided that details of the new co-ordinate system would be published in the form of a note in various astronomical journals. 